\title{
Urgences
}

\section{Lindberg : de la modernité dans " l'air "}

\section{Bruno Roy}

Numéro 26, décembre 1989

Des textes qui chantent

URI : https://id.erudit.org/iderudit/025552ar

DOI : https://doi.org/10.7202/025552ar

Aller au sommaire du numéro

Éditeur(s)

Urgences

ISSN

0226-9554 (imprimé)

1927-3924 (numérique)

Découvrir la revue

Citer cet article

Roy, B. (1989). Lindberg : de la modernité dans " l'air ". Urgences, (26), 28-38. https://doi.org/10.7202/025552ar d'utilisation que vous pouvez consulter en ligne.

https://apropos.erudit.org/fr/usagers/politique-dutilisation/ 


\section{Lindberg: de la modernité dans «l'air » Bruno Roy}

\section{Lindberg !}

Des hélices: astro-jets - whisper-jets - clipper-jets

Turbos, à propos:

Chu pas rendu chez Sophie

Qui a pris l'avion St-Esprit

5 de Duplessis

Sans m'avertir

Alors chu parti

Sur Québec Air, Transworld, Northern, Eastern, Western Pi Pan American!

Mais ché'pu...

Oh chu rendu.

J'ai été:

Au Sud du Sud

Au soleil bleu blanc rouge

15 Les palmiers et les cocotiers glacés

Dans les pôles aux esquimaux bronzés

Qui tricotent des ceintures fléchées farcies

Et toujours la Sophie

Qui venait de partir.

20 Alors chu r'parti

Sur Québec Air, Transworld, Northern, Eastern, Western

Pi Pan American!

Mais ché'pu...

Oh chu rendu.

$25 Y$ avait même une compagnie qui engageait

Des pigeons qui volaient en dedans

Et qui faisaient le balan

Pour la tenir dans le vent

C'était absolument, absolument, absolument

30 Très salissant.

Alors chu r'parti

Sur Québec Air, Transworld, Northern, Eastern, Western

Québec Air, Transworld, Northern, Eastern, Western

Pi Pan American!

Ma Sophie a pris une compagnie 
Qui volait sur des tapis de Turquie

C'est plus parti. Et moi à propos

Chu rendu à dos de chameau...

Je préfère

Pi Pan American!

Mais je ne sais plus où je suis rendu

Pi j'ai fait une chute, une kriss de chute, en parachute

Et j'ai retrouvé ma Sophie

45 Elle était dans mon lit

Avec mon meilleur ami,

Et surtout

Mon pot de biscuits

Que j'avais ramassé

50 Sur Québec Air, Transworld, Northern, Eastern, Western

Et Pan American.

Paroles de Claude Péloquin Musique de Robert Charlebois

C'est connu, Robert Charlebois n'a pas souvent travaillé seul. Nombre de paroliers ont accompagné ou suscité sa démarche artistique: Mouffe, Marcel Sabourin, Réjean Ducharme, Claude Péloquin et bien d'autres. C'est avec la collaboration de ce dernier et celle des musiciens du Jazz libre, en ajoutant la voix de Louise Forestier, qu'il créa le premier succès contreculturel de la chanson québécoise: Lindberg. Il faut retenir, de cette époque, que c'est sa période électrisante la plus criée. $\mathrm{Ce}$ qui contrastait avec les chansons de ses deux premiers albums, qui sont très écrites. Avec Lindberg (et tout son quatrième album), Charlebois rompt avec le "chansonnier correct». Il troque son habit rayé de débutant pour des «oripeaux psychédéliques de chanteur populaire ${ }^{1}$. La double rupture avec lui-même (ses premières chansons) et un genre (le chansonnier) amorça avec Lindberg, California, C.P.R. Blues, Egg Generation sa montée vers un type de chansons qui allait redéfinit le genre. La musique descendait directement des "rythm and blues", inspirée de certains groupes américains tels les Mothers of Invention ou les Cream. L'élément proprement québécois fera la différence, mais jamais l'élément

1 Jacques Julien, Robert Charlebois, l'enjeu d'a Ordinaire ", Montréal, Triptyque, 1987, p. 28. 
30

délire ne sera absent. Robert Charlebois, écrira Jacques Julien, dans un livre qu'il lui consacre, "s'est trouvé mêlé à un groupe de créateurs féconds et bien enracinés dans l'éclatement général de la fin des années 60 *2.

La chanson québécoise est apparue à l'époque des grands bouleversements sociaux qu'a apportés la Révolution tranquille. C'est cependant un spectacle qui détermina un avant et un après de la chanson moderne au Québec: L'Osstidshow. Ce spectacle fut présenté au théâtre de Quat'Sous par Robert Charlebois, Louise Forestier, Mouffe, Yvon Deschamps, le Quatuor du Nouveau Jazz libre du québec et l'organiste Jacques Perron. La première eut lieu le 2 mai 1968. Spectacle de conception, sorte de "happening " culturel, la formule intégrait chansons, monologues, sketches, musique et offrait au spectateur une manière de décloisonnement des arts de la scène. Les chansons de son quatrième album proviennent de ce spectacle ouvert à la productivité totale, s'inscrivant dans les "happenings" théâtraux de l'époque qui favorisaient la "folie totale». Lindberg, par exemple, instaure le temps du rêve fou, du voyage multiple. Sa musique est faite de mouvements pluridimensionnels, sans causes et effets. Lindberg décrit la perte de l'individualité dépersonnalisée, aliénée:

Mais je ne sais plus où je suis rendu

Pi j'ai fait une chute, une kriss de chute, en parachute

Et j'ai retrouvé ma Sophie

Elle était dans mon lit

Avec mon meilleur ami,

Et surtout mon pot de biscuits

Que j'avais ramassé

Sur Québec Air [...]

Ces paroles sont venues d'un défi entre Robert Charlebois et Claude Péloquin. Ce dernier, écrivain, écoutait le chanteur lui expliquer qu'il était difficile de composer une bonne chanson populaire. Non seulement ils s'exécutèrent rapidement, mais ils gagnèrent respectivement leur défi. Louise Forestier rappelle les circonstances:

Tout s'est enchaîné, tout s'est développé, si bien qu'à un moment donné je me retrouvai dans un appartement avec Robert, Mouffe, Péloquin et Sophie. Ce genre commune a 
duré trois mois, un temps suffisant pour créer de bonnes choses. Un soir Charlebois avait défié Pélo d'écrire les paroles d'une chanson en cinq minutes. Pélo avait accepté, il était monté aux chambres et cinq minutes plus tard il redescendit avec $\propto$ Lindberg $\nsim$. Robert composa la musique, en cinq minutes. C'était en janvier 1968. ${ }^{3}$

C'est ainsi qu'est née au Québec, écrit Diane Gagnon, «la première chanson psychédélique " 4 , laquelle fut enregistrée pour la première fois sur disque Gamma (GS 120) qui contenait le matériel de L'Osstidshow et qui a pour titre Robert Charlebois avec Louise Forestier. Chose rare pour l'époque ${ }^{5}$, les textes des chansons étaient inclus dans la pochette dont la conception graphique est de Robert Barbeau et la photo de l'endos de Robert Poly. Quant à l'accompagnement musical du disque, en plus du Quatuor du Nouveau Jazz du Québec, on note la présence de Philippe Gagnon, le violonneux électrique, et Michel Robidoux, raccordeur musical. Subitement, dans ce disque, les formes musicales et littéraires disparaissaient au profit de mélodies dissonantes accompagnées de mots apparemment sans liens logiques. Lindberg et autres chansons du même "air " furent perçues comme des excès de langage, perçues aussi comme une provocation: la manière de traiter le texte poétique.

Certes, une véritable musique psychédélique proprement québécoise et qu'a amorcée Lindberg n'est pas venue seule. Le contexte culturel et social est soutenu de discours pluralistes et multiples, tant sur la langue que sur la société elle-même. Il y a une relation réelle des gens avec leur culture. Au théâtre, la fin des années 60 impose une redéfinition de l'imaginaire québécois: Les belles-sœurs de Michel Tremblay (1968) et Le Cid maghané de Réjean Ducharme (1968), Double jeu de Françoise Loranger (1969). Le roman traçait déjà les lignes d'un nouveau territoire culturel: Le cassé de Jacques Renaud (1964), Salut Galarneau! de Jacques Godbout (1967). C'est aussi en 1968 que Pierre Vallières fait paraître Negres blancs d'Amérique. À la radio, CJMS, sur la base du " hit parade», devient la station des jeunes dont c'est désormais l'identité.

3 Benoît L'Herbier, Charlebois, qui es-tu ?, Montréal, Éd de l'Homme, 1971, p. 80 .

4 Diane Gagnon, Photo-Vedettes, $1^{\text {er }}$ aout 1970, p. 5.

5 La règle de trois minutes pour une chanson sera outrancièrement battue. Lindberg fait exactement 5 min $23 \mathrm{~s}$. 
Dans le domaine de la musique, le Quatuor du Nouveau Jazz libre du Québec, puis l'Infonie (Raoul Duguay), tout en participant aux diverses représentations de L'Osstidshow, partagèrent l'esprit de libération qui souffla sur le Québec. Dans ces mêmes années, on assistait à la montée de l'élite politique francophone à Ottawa, incarnée par «trois colombes»: Jean Marchand, Gérard Pelletier et Pierre-Elliott Trudeau. Au Québec, René Lévesque se préparait à fonder le Parti québécois, qui réunirait l'ensemble des forces nationalistes.

Quant au contexte plus spécifiquement contre-culturel, la jeunesse québécoise suivait le mouvement de la contreculture, mais en retrait. C'est que la musique dite underground, perçue comme un moyen de traduire les expériences psychologiques, repose encore, à la fin des années 1960, sur une certaine clandestinité. En parallèle, principalement avec Mainmise, s'est développée par la suite une presse underground qui "charge" sur les mêmes réalités. Les mêmes thèmes recoupent des manières analogues : imagination délirante, révolte contre les interdits, voyages multiples, etc. Voilà comment, par leur goût pour l'irrationnel, des chansons comme Lindberg appartiennent à leur époque.

Sur la Place des Nations (en 1970), la foule lévite. Elle lévite avec « Lindbergh $\approx$. Tout le monde chante. Les caméras tournent sur le pivot comme des folles. Les projecteurs des grandes tours balaient la foule et des grands $\propto V \propto$ trouent le ciel. La foule hurle $\propto \mathrm{Pi}$ chu parti $\nsim$. Tout le monde est en l'air. ${ }^{7}$

L'effet de balancement est indiqué, dans la chanson, par un retour constant d'une montée/chute: telle est la ligne musicale de Lindberg. Le traitement de l'isotopie «voyage", par exemple, s'inscrit dans une perspective "surréalisante " et les sèmes communs sont utilisés à des fins ironiques, certes, mais peut-être davantage à des fins de liberté totale. La "surréalité" s'organise autour de l'aire sémantique du

6 Georges Kahl écrit Lindbergh avec un $h$ alors que dans le texte de Claude Péloquin (celui de la pochette), il n'y en a pas. Lindbergh, c'est l'aviateur américain qui, en 1927, traversa l'Atlantique avec son a Spirit of Saint-Louis ". II fut le premier à réussir la liaison aérienne sans escale de New York à Paris. Lindberg, la chanson, c'est Sophie a qui a pris l'avion St-Esprit de Duplessis sans m'avertir"... Certes, les mots sont en relation d'inter-référence. Ils mettent surtout en évidence le jeu totalement gratuit des a sens ".

7 Georges Kahl, Le devoir, 8 septembre 1970, p. 13. 
sème «parti»: partir (déplacement), pas mal parti (trip), s'envoyer en l'air, être «high , telle était la disposition des gens de l'époque. Lindberg, littéralement, c'est le voyage dans tous les sens, le voyage de tous les sens. Drogues et érotisme en sont les pôles.

Ma Sophie a pris une compagnie
Qui volait sur des tapis de Turquie
C'est plus parti
[...]
Et j'ai retrouvé ma Sophie
Elle était dans mon lit
Avec mon meilleur ami

Quant aux termes mêmes de la chanson, ils appartiennent à l'isotopie du vol. Ils suggèrent une hyperbole absurde, pạr une mise en abyme qui indique deux plans sémantiques:

$Y$ ' avait même une compagnie qui engageait

Des pigeons qui volaient en dedans

Et qui faisaient le balan

Pour la tenir dans le vent

(v. 25-28)

À l'ordre contigu de la représentation du réel, se substituent dans Lindberg les sursauts analogiques du délire. Le monde à l'envers? Pourtant, les figures de renversement appartiennent à un topos culturel bien québécois. Péloquin s'y réfere abondamment à l'aide d'une figure de rhétorique appelée l'oxymoron:

Au soleil bleu blanc rouge

Les palmiers et les cocotiers glacés

Dans les pôles aux esquimaux bronzés

Qui tricotent des ceintures fléchées farcies

Depuis Lindberg, le spectacle de la chanson semble voué à l'empirisme surréaliste dont le trait s'associe à la fantaisie verbale fréquentant l'humour. "Briseur de syntaxe et de logique ", pour reprendre la critique, la chanson du trio Poliquin/Charlebois/Forestier multiplie les écarts qui vont à l'encontre d'une norme de cohérence du discours et d'une norme esthétique. Lindberg nous apprend le jeu verbal par le clin d'œil. Jusque là, la chanson poétique avait tellement été sérieuse, de ce sérieux qui en faisait une chose sacrée.

Il est vrai que la chanson québécoise, soutenue par un discours idéologique qui ne pouvait lui échapper, montrait depuis 1960 une rare force d'expression collective et le phéno- 
34

mène joual en exprima les éléments les plus radicaux. Il était l'expression d'une bâtardise linguistique que la chanson assumait dans un contexte de redéfinition des codes culturels. Lindberg, par exemple, a constitué sans l'ombre d'un doute la violation d'une norme instituee, celle des chansonniers, celle d'une chanson poétique en accord avec une vision du * pays *. Voilà comment cette chanson est allée à l'encontre d'une norme esthétique. Ainsi, dans Lindberg, si le sacre est une adjonction (fond sonore de la chanson, la voix de Louise Forestier) qui s'actualise en valeur sémantique ${ }^{8}$, au plän sonore, le sifflement des fricatives (hostie, sacristie, Christ) rend le rythme plus agissant. L'agrément des consonances, par exemple, permet le jeu verbal et le calembour. De plus, la pratique rythmique est en rupture avec le modèle français du poème versifié. Le texte de Péloquin renonce à la métrique, amplifiant plutôt le caractère accentuel de la langue française.

À l'inverse du poème versifié, ce texte ne peut pas tenir de la notion de rythme, dans son sens de périodicité. En effet, dans Lindberg, le retour à l'accent est très irrégulier, phénomène qui rapproche les paroles de Péloquin de la chaîne parlée. La métrique, ici, repose sur des accents de mots forts et fixes ou égaux. Lindberg annonce un trait sonore caractéristique que Charlebois conservera. Chez lui, on retrouve « un retour constant d'un accent de hauteur sur les accents de mot primaire et secondaire, par une remontée de la voix, plus ou moins à la même hauteur et suivant des écarts étroits, forts ou égaux " ${ }^{9}$. D’autres forces sonores que la mélodie s'imposent.

Dans la langue chantée de Charlebois et, plus tard, des groupes québécois, on retrouve une régularité du mouvement rythmique. Ce mouvement peut être comparé à celui de l'anglais, grâce à cet effet de balancement (accent secondaire) appelé «wave intonation * et qui serait typiquement américain, comme dans ces mots: laboratory, continuation, etc. Ainsi, la

8 Le sacre est encore une manifestation que l'on contourne. Louise Forestier raconte (le 29 juin 1987 a Radio-Québec) que pour l'enregistrement d'une émission - $\alpha$ et cela pour que ça passe a la radio " - il a fallu enregistrer a triste " et non a Christ". Nous sommes loin de cette époque où Louise Forestier habillée en curé prononçait ses « Kriss « devant un auditoire réceptif. Le joual et tout ce qu'il a charrié de contestataire à l'époque n'est évidemment plus glorifié aujourd'hui.

9 Desdémone Bardin, Culture du rythme, culture du verbe, thèse, Paris, Université de Paris VI, Département de recherches linguistiques, pp. 42-45. 
forme linguistique de Lindberg est manifestée par une substance rythmique dont la structure peut être dite iambique par une suite de stimulations données:

Des hélices: astro-jets - whisper-jets - clipper-jets

Turbos, à propos:

Chu pas rendu chez Sophie

Qui a pris l'avion St-Esprit

de Duplessis

Sans m'avertir

Les unités rythmiques de ce refrain, bien qu'appartenant à une structure binaire, sont différentes du rythme de la marche où c'est le premier temps qui est accentué. L'accentuation et le mouvement "rythmico-intonatif" tendent à se distinguer du mouvement rythmique du français dit $\propto$ international ». Ici, le joual se prête différemment (plus facilement?) à la musique et aux effets sémantiques qui en résultent. La ligne mélodique de Lindberg se caractérise par son balancement dû à un retour fréquent d'accentuation:

Alors chu parti sur

Québec Air, Transworld, Northern, Eastern, Western

Pi Pan American!

Depuis Charlebois, ce qui caractérise la chanson québécoise, c'est l'abandon d'une alngue littéraire au profit d'une langue populaire. On a perçu ce changement comme une violation, mais cette violation ne pouvait demeurer sans effet rhétorique pour elle-même. Au Québec, la musique populaire est plus nord-américaine que jamais et $L$ indberg en était lillustration la plus populaire. Le joual qui s'y trouvait révéla une intonation plus ou moins américanisée de la phrase chantée. Au centre de ce phénomène linguistique: la diphtongaison.

Au point de vue du joual, les phénomènes de transformations altèrent la constitution phonique du mot comme ils altèrent, conséquemmemt, sa représentation graphique. Cette altération concerne la morphologie du mot

mais ché' pu

Oh chu rendu 10

10 lci, le * oh" et le * où " sont indécidables dans la vocalise. II faut les voir écrits dans le texte qui accompagne la pochette (lequel est intégralement reproduit dans le livre de Lucien Rioux, Robert Charlebois, coll. a Poésie et 
Dans cet extrait, on assiste à une compression-décompression qui s'incarne dans la diphtongue. L'écrasement de la syllabe (sa réduction) produit une différence de volume et de durée de la syllabe qui marque la différence de répartition de l'énergie articulatoire entre le français standard et la langue populaire. Le joual, ici, a recours aux procédés d'altération de la langue commune dont le trait collectif est la diphtongaison qui est une forme systématique de l'allongement itératif: moé-même, noères, bêêtes, etc. La diphtongaison tente de rétablir l'équilibre rythmique par l'allongement vocalique accusant une prépondérance des nasales. Dans Lindberg, le mot peut être vu comme un phénomène essentiellement phonétique.

Plus intéressant encore, la diphtongaison pourrait être associée au phénomène de «syncopation *, pris dans son sens musical: procédé rythmique qui consiste à déplacer, en le prolongeant, un temps fort sur un temps faible. Nous assistons donc à l'émission anticipée d'une note qui est ainsi attaquée entre deux temps. Cette sorte d'élan pris pour la production d'un temps fort correspond, dans le texte chanté, au phénomène d'accentuation dont nous parlions plus haut. Ainsi, "ché » sert de point d'appui pour la production d'énergie accentuelle de la syllabe « pu : « Mais ché puuuuu... *

De plus, les syllabes "pu " et «rendu * marquent une prépondérance des nasales que la musique amplifie. On peut, en effet, transcrire l'effet obtenu comme un allongement itératif. La parole, transfigurée par le son, laisse l'intonation se poursuivre comme l'écho. Quant au *Mais *, il a un effet de regain, alors que le « $\mathrm{Oh}$ » est une suppression complète du " où». La figure, ici la parataxe, joue sur la syntaxe. L'opération de substitution perturbe la sémantique. En remplaçant un mot par un autre, l'auteur nous amène au plan du contenu. Nous sommes en présence d'une antithèse et d'une inversion stylistique:

Mais ché' pu
Oh chu rendu
Mais je ne sais plus

Où je suis rendu

Le mouvement de cet aller retour linguistique permet une très grande efficacité sémantique. En effet, dans Lindberg, le degré zéro, c'est la langue populaire; c'est * ché' pu oh chu rendu *. Par * Mais je ne sais plus où je suis rendu *, la langue

chansons", Paris, Seghers, 1973). Leur seule audition est insuffisante pour identifier distinctement les phonemes. 
correcte constitue plutôt un écart, linguistique et sémantique. L'auteur utilise comme figure une suppression négative engendrant par son antiphrase une antithèse stylistique puisque, précisément, la représentation mentale du vers est modifiée. Il y a donc une double altération: sur la sémantique, sur la logique. Or, à propos du texte de Péloquin, il y a figure sémantique parce qu'il y a infraction au code lexical qui se donne à lire: Oh chu rendu/où je suis rendu. Ce qui domine, on le voit mieux maintenant, c'est le mouvement pulsionnel du texte qui lui donne sa "rythmicité" américaine.

Le spectacle de L'Osstidshow, auquel appartient Lindberg, a modifié le discours chansonnier (procès énonciatif); un trait, par exemple, l'élément consonant est disparu au profit de l'élément dissonant. La voix contrôlée du chansonnier cède la place à celle, plus criée, de la période psychédélique. L'œuvre majeure de Charlebois demeure l'invention d'un comportement scénique relevant de la performance. Là, le texte devient théâtre: textes et musiques se jouent hors de la continuité linéaire. Comme dans Lindberg, l'accent devient un choix esthétique, c'est-à-dire un travail artistique qui intéressera, plus tard, les groupes québécois. Car la langue de L'Osstidshow sous-tend, en dernière analyse, une recherche d'identité.

Depuis Lindberg, lidée d'une nouvelle stylistique de la chanson est inséparable d'une nouvelle rhétorique du texte chanté. Certes, Charlebois et ses paroliers ont expérimenté des «brisures linguistiques " qu'il ne faut pas confondre avec une rupture totale de la langue. Dans la chanson québécoise, Lindberg a amorcé l'histoire des seuils plus ou moins transgressifs de notre langue, mais cette chanson, comme d'autres productions culturelles, n'a pas créé le problème du joual, elle a permis l'expression d'une collectivité en apprivoisant la culture de son temps.

Avec Lindberg, est venue au Québec une chanson tout court. Le succès de cette chanson a eu le mérite d'avoir réalisé une jonction entre la chanson québécoise et la chanson populaire, c'est-à-dire entre la chanson plus traditionnelle et le rock. D'aucuns pensent que c'est Robert Charlebois qui contribua à mener la chanson québécoise à son état présent. Lindberg, en effet, installa la notion pop dans le domaine artistique québécois. La musique pop est à la jonction de deux tendances: yéyé et chansonnier, sur lesquelles est venue se greffer la forme moderne de la chanson et à travers laquelle les groupes québécois allaient naturellement se reconnaître. 
38

Lindberg a débordé la chanson elle-même, parce que le texte a mis en jeu le sens d'une langue, d'une culture, d'une identité. Elle a reconsidéré les modes de « signifier» de toute une société. Lindberg, vue comme une rhétorique de notre modernité, voire de notre américanité, nous a fait passer de la chanson française à la chanson québécoise. Lindberg indique, pour son époque, le temps présent. Survolant l'Amérique québécoise, cette chanson est la première métaphore transgressive de notre identité originelle. Les nouvelles fomes de chansons qui ont suivi ce premier "envol" sont vues, désormais, comme des symboles de notre civilisation moderne. 